\title{
High-sensitivity high-resolution X-ray imaging with soft-sintered metal halide perovskites
}

\author{
Sarah Deumel ${ }^{1,2 凶}$, Albert van Breemen ${ }^{3}$, Gerwin Gelinck ${ }^{3,4}$, Bart Peeters ${ }^{3}$, Joris Maas ${ }^{3}$, \\ Roy Verbeek ${ }^{3}$, Santhosh Shanmugam ${ }^{3}$, Hylke Akkerman ${ }^{3}$, Eric Meulenkamp ${ }^{3}$, Judith E. Huerdler', \\ Manognya Acharya ${ }^{1}$, Marisé García-Batlle ${ }^{5}$, Osbel Almora ${ }^{5}$, Antonio Guerrero ${ }^{5}$, \\ Germà Garcia-Belmonte $\mathbb{1}^{5}$, Wolfgang Heiss $\mathbb{1}^{2}$, Oliver Schmidt' and Sandro F. Tedde ${ }^{1}{ }^{1 凶}$
}

To realize the potential of artificial intelligence in medical imaging, improvements in imaging capabilities are required, as well as advances in computing power and algorithms. Hybrid inorganic-organic metal halide perovskites, such as methylammonium lead triiodide $\left(\mathrm{MAPbl}_{3}\right)$, offer strong X-ray absorption, high carrier mobilities $(\mu)$ and long carrier lifetimes $(\tau)$, and they are promising materials for use in X-ray imaging. However, their incorporation into pixelated sensing arrays remains challenging. Here we show that X-ray flat-panel detector arrays based on microcrystalline MAPbl $_{3}$ can be created using a two-step manufacturing process. Our approach is based on the mechanical soft sintering of a freestanding absorber layer and the subsequent integration of this layer on a pixelated backplane. Freestanding microcrystalline $\mathrm{MAPbl}_{3} \mathrm{wafers}$ exhibit a sensitivity of $9,300 \mu \mathrm{C} \mathrm{Gy}_{\text {air }}^{-1} \mathrm{~cm}^{-2}$ with a $\mu \tau$ product of $4 \times 10^{-4} \mathrm{~cm}^{2} \mathrm{~V}^{-1}$, and the resulting $\mathrm{X}$-ray imaging detector, which has 508 pixels per inch, combines a high spatial resolution of 6 line pairs per millimetre with a low detection limit of 0.22 nGy $\mathbf{y}_{\text {air }}$ per frame.

T he use of artificial intelligence (AI) in medical imaging is steadily growing ${ }^{1-3}$. Currently, task-specific AI applications are able to match and occasionally exceed human intelligence, and it has been predicted that AI will surpass the skills of a radiologist in the next 50 years ${ }^{4}$. The development of such technology requires advances in computing power and algorithms, but also advances in curation and imaging capabilities. X-ray detectors with increased resolution can, in particular, provide improved medical images that further enhance the benefits of AI technologies ${ }^{5}$. X-ray detectors can be divided into two main classes: ${ }^{6-8}$ indirect-conversion detectors and direct-conversion detectors. Indirect converters exhibit high sensitivity but suffer from low spatial resolution'. Direct converters can capture high-resolution images (up to 10-15line pairs per millimetre $\left(\mathrm{lp} \mathrm{mm}^{-1}\right)$ ) but at a relatively high applied electric field $\left(1-10{\mathrm{~V} \mu \mathrm{m}^{-1}}^{2}\right.$ and relatively low sensitivity ${ }^{10-14}$. The requirement is an X-ray detector that combines high resolution (to enhance AI performance) with high sensitivity (to reduce patient X-ray dose).

Hybrid inorganic-organic perovskites, such as methylammonium lead iodide $\left(\mathrm{CH}_{3} \mathrm{NH}_{3} \mathrm{PbI}_{3}\right.$, abbreviated as $\mathrm{MAPbI}$ ), offer high electron and hole diffusion lengths due to their high charge carrier mobilities $(\mu)$ and long carrier lifetimes $(\tau)$, and have demonstrated promising characteristics for use as direct X-ray converters ${ }^{15-21}$. For example, polycrystalline and single-crystalline hybrid inorganic-organic perovskites have exhibited a $\mu \tau$ product of $2.0 \times 10^{-4}$ and $1.2 \times 10^{-2} \mathrm{~cm}^{2} \mathrm{~V}^{-1}$, respectively, which are in the same range as polycrystalline cadmium zinc telluride (CZT) $)^{13,15-21}$. The high X-ray absorption coefficient of $\mathrm{MAPbI}_{3}$ over large parts of the energy spectrum used in healthcare also makes it an ideal candidate for use in next-generation imaging systems. However, the integration of direct X-ray converter layers onto a pixelated electrode substrate (often referred to as a backplane), which converts the generated $\mathrm{X}$-ray signal of each pixel into a two-dimensional digital image, can be challenging from a manufacturing perspective. Thus, while X-ray detection with direct-conversion perovskites has been demonstrated, the integration of perovskite detecting layers with pixelated backplanes has only received limited attention ${ }^{17,22}$.

In this Article, we report a two-step manufacturing process to create X-ray flat-panel detectors that combine high spatial resolution and high sensitivity. Our approach separates the fabrication of an X-ray absorber layer (which is made of microcrystalline $\mathrm{MAPbI}_{3}$ ) with a thickness of several hundreds of micrometres from integration onto the backplane. The integration is subsequently performed at room temperature, and thus, backplane limitations regarding the temperature budget are not a factor. Our approach also allows for the independent optimization of the $\mathrm{MAPbI}_{3}$ absorber formation.

\section{X-ray imager architecture}

The layer stack of the imaging X-ray detector is shown in Fig. 1a. The glass-based backplane is a self-aligned dual-gate indium gallium zinc oxide thin-film transistor array, which is described and characterized elsewhere ${ }^{23,24}$. The range of applicable voltages on the backplane is limited due the missing high-voltage protection of the pixels. Bottom electrodes are made of a molybdenum-chromium alloy. On top of the backplane, a grid structure made of an approximately $10-\mu \mathrm{m}$-thick photoresist is used as a mechanical anchoring structure for the thick absorber layer. Without this grid, the mechanical adhesion between $\mathrm{MAPbI}_{3}$ and backplane was found to be poor, indicated by the release of the wafer after a few days. However, with the grid, no spontaneous detachment can be seen in several months. Pull tests revealed a tensile strength of $100 \mathrm{mN} \mathrm{mm}^{-2}$. The grid is

'Siemens Healthineers AG, Technology Excellence, Erlangen, Germany. ${ }^{2}$ Institute Materials for Electronics and Energy Technology (i-MEET), Department of Materials Science and Engineering, Friedrich-Alexander-Universität, Erlangen-Nürnberg, Energy Campus Nürnberg, Nuremberg, Germany. ${ }^{3} \mathrm{Holst}$ CentreTNO, The Netherlands Organization for Applied Scientific Research, Eindhoven, The Netherlands. ${ }^{4}$ Molecular Materials and Nanosystems, Institute of Complex Molecular Systems, Eindhoven University of Technology, Eindhoven, The Netherlands. ${ }^{5}$ nstitute of Advanced Materials (INAM), Universitat Jaume I, Castelló, Spain.凶e-mail: sarah.deumel@siemens-healthineers.com; Sandro.tedde@siemens-healthineers.com 
$\mathbf{a}$

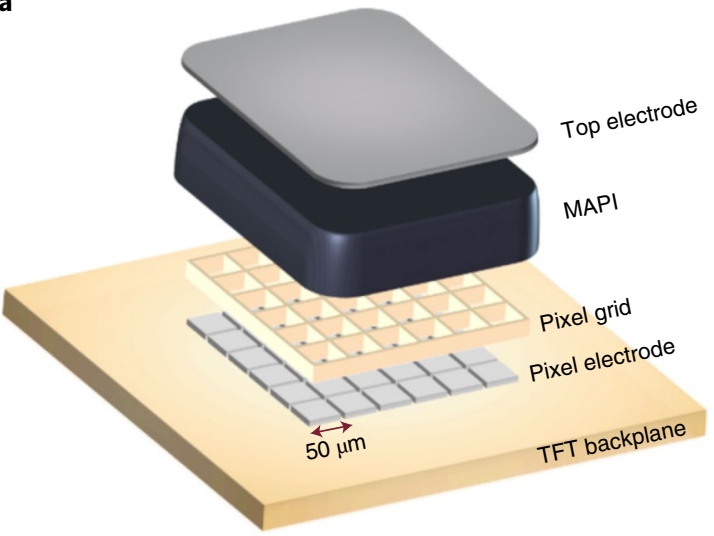

b

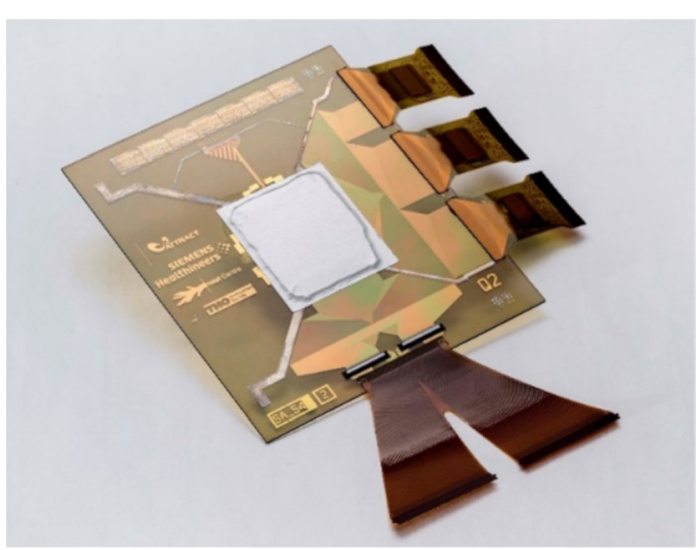

d

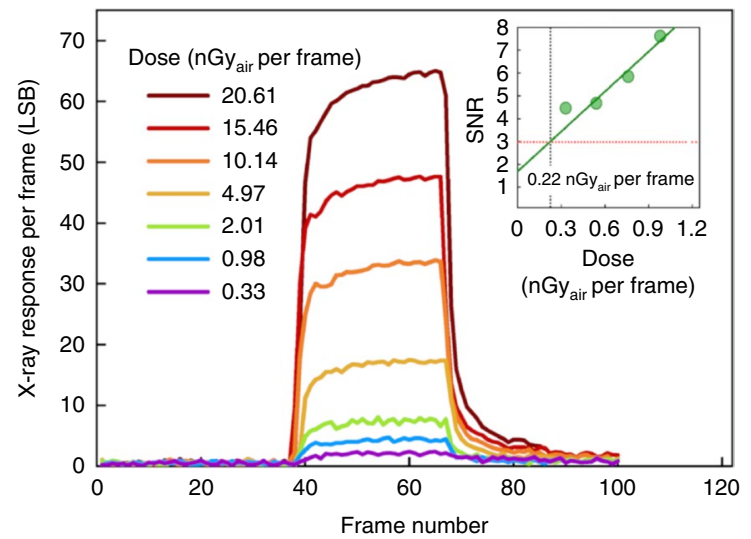

Fig. 1 | Perovskite X-ray imaging detector. a, Exploded view of the different elements of the X-ray imager. $\mathbf{b}$, Photograph of the final detector with chip-on-glass gate driver integrated circuits (bottom part) and chip-on-flex ROIC (right side); the MAPbl ${ }_{3}$ (MAPI) layer attached on the backplane is encapsulated by a laminated metallic foil (centre); and flex bonds connect the array with the reading and driving printed circuit boards. The glass substrate

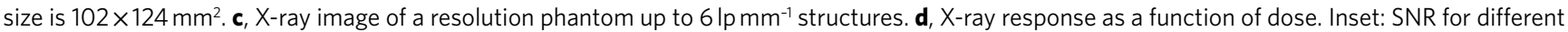
pulses with a detection limit of $0.22 \mathrm{nGy}_{\text {air }}$ per frame. Each frame is $35 \mathrm{~ms}$.

filled with liquid $\mathrm{MAPbI}_{3}$ that acts-after its recrystallization-as an adhesion promoter for the attachment of the X-ray absorber, which consists of a $230-\mu \mathrm{m}$-thick $\mathrm{MAPbI}_{3}$ layer; this thickness was chosen with respect to the limited applicable bias voltage at the imager. As the cathode, a chromium (Cr) layer is deposited on top of $\mathrm{MAPbI}_{3}$. The active area of the imager is encapsulated with a barrier foil to avoid environmental influences, as shown in Fig. 1 b.

This direct-conversion X-ray detector can capture objects with a very high resolution of $6 \mathrm{lp} \mathrm{mm}^{-1}$ (Fig. 1c) and shows an unprecedented low detection limit of $0.22 \mathrm{nGy}_{\text {air }}$ per frame at an applied

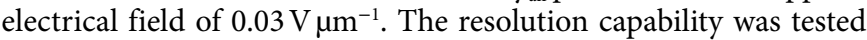
by $\mathrm{X}$-ray imaging a phantom having structures made of three lines at different spacings of 5.0, 5.5 and $6.0 \mathrm{lp} \mathrm{mm}^{-1}$. The detection limit is calculated taking-for each frame-the average signal of pixels from a region of interest (ROI) for a 1-s-long exposure at different doses and considering a signal-to-noise ratio (SNR) of $>3$, as shown in Fig. $1 \mathrm{~d}^{25}$. The beam quality was generated using an X-ray source with an anode bias of 70 peak kilovoltage $(\mathrm{kVp})$, and filtering the resulting radiation with $21 \mathrm{~mm}$ aluminium (Al) and additional attenuation with $1 \mathrm{~mm} \mathrm{~Pb}$ (Supplementary Information provides details about the X-ray setup). The signal is presented in the least significant bit (LSB), the smallest possible unit of the analogue-to-digital converter in the ROIC, which-in the used settings-is equivalent to 48 electrons.

The dose per frame was varied between 0.33 and $20.61 \mathrm{nGy}_{\text {air }}$, and the SNR was determined from the resulting height $(H)$ of the pulses and variance of the background noise $(h)$ according to the following equation: $\mathrm{SNR}=2 \mathrm{H} / h \geq 3$ (ref. ${ }^{25}$ ). The SNR is inversely proportional to the dose (Fig. $1 \mathrm{~d}$, inset) and can be linearly fitted, which leads to a dose of $0.22 \mathrm{nGy}_{\text {air }}$ per frame at $\mathrm{SNR}=3$. This is due to the impressive stability of the mean value of the ROI in the range of $0-2$ LSB in the observed period. Electrical stability is reached after $90 \mathrm{~min}$ of biasing of the imager at an electrical field of $0.03 \mathrm{~V} \mathrm{\mu m}^{-1}$, which is the time required to achieve an almost ionic equilibrium state (Supplementary Fig. 1a). With a frame rate of 28.6 frames per second, the detection limit is $6.3 \mathrm{nGy}_{\text {air }} \mathrm{s}^{-1}$, which is $20 \%$ lower compared with the best reported detection limit of perovskites: Wei and co-workers made a 1-mm-thick single crystal of methylammonium lead tribromide $\left(\mathrm{MAPbBr}_{3}\right)$ alloyed with $\mathrm{Cl}$, achieving the lowest detectable dose rate of $7.6 \mathrm{nGy}_{\text {air }} \mathrm{s}^{-1}$ for a photon energy of $8 \mathrm{keV}$ (ref. ${ }^{26}$ ). Previously reported limits for $\mathrm{MAPbI}_{3}$ are even higher: $19.1 \mu \mathrm{Gy}_{\text {air }} \mathrm{s}^{-1}$ for single-crystal $\mathrm{MAPbI}_{3}$ (ref. ${ }^{19}$ ). With A-site cation variation, Huang et al. could reduce the detection limit to $16.9 \mathrm{nGy}_{\text {air }} \mathrm{s}^{-1}$ (ref. ${ }^{20}$ ).

\section{Two-step manufacturing process}

The manufacturing process of our perovskite X-ray detector consists of two phases: the first focuses on the production of the X-ray absorber layer. We chose a mechanical soft-sintering process, which is described in detail in ref. ${ }^{18}$. The used microcrystalline $\mathrm{MAPbI}_{3}$ powder is commercially available, where the grain size varies between 0.1 and $100.0 \mu \mathrm{m}$, whereas $85 \%$ of the measured sizes are 
a

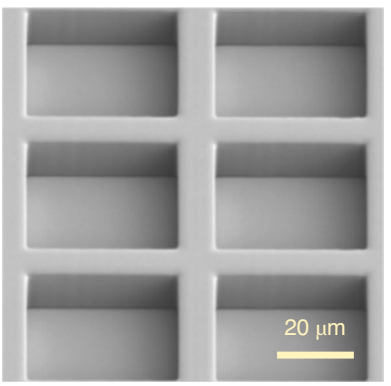

d

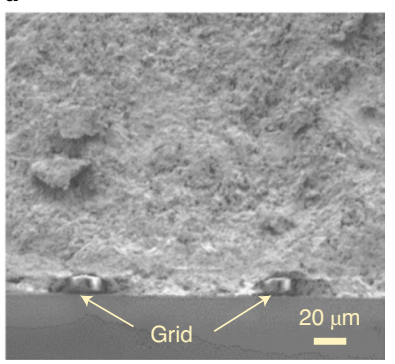

b

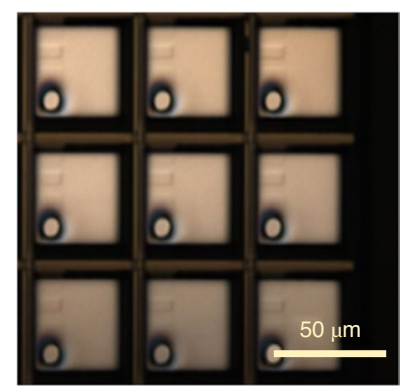

e

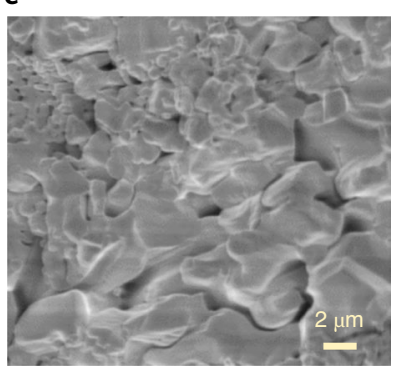

c

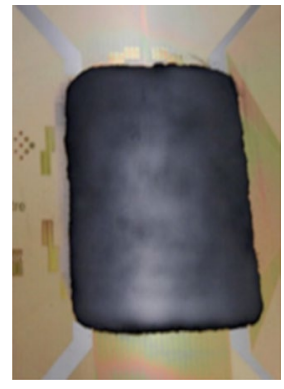

f

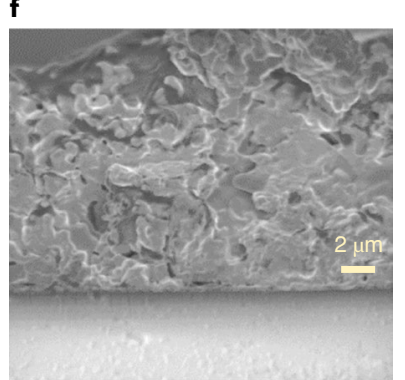

Fig. 2 | Characterization of the manufacturing process. a, SEM image of the grid photoresist structure. The bird's eye view shows the regular shape of the grid. The width of the structure is $10 \mu \mathrm{m}$. The pixel pitch is $50 \mu \mathrm{m}$. b. Optical microscopy image of the grid structure. The bright areas are the bottom electrodes defining the pixel size. $\mathbf{c}$, Photograph of the $\mathrm{MAPbl}_{3}$ absorber layer attached to the backplane. The size of the $\mathrm{MAPbl}_{3}$ absorber layer is $3 \times 4 \mathrm{~cm}^{2}$ and fully covers the area of the electrode array. d-f, Cross-sectional SEM images of different MAPbl $_{3}$ layers: bottom part of an 880 - $\mu$ m-thick absorber layer above an indium tin oxide glass with the photoresist grid visible and indicated with arrows (d); a closer look into the wafer shows grains with sizes between 0.5 and $5.0 \mu \mathrm{m}$ and pores between them (e); grains of the recrystallized $\mathrm{MAPbl}_{3}$ layer with smaller grains up to $2 \mu \mathrm{m}$ (f).

below $5.0 \mu \mathrm{m}$ (Supplementary Fig. 2a,b). For the investigation of the structural properties of the powder, an X-ray diffraction measurement has been performed (Supplementary Fig. 2c). The mean diffraction peaks at $14.1^{\circ}, 23.4^{\circ}, 24.5^{\circ}, 28.1^{\circ}$ and $28.4^{\circ}$ are related to the lattice planes (110), (211), (202), (004) and (220), respectively, and fit well with the values of the tetragonal phase with the $I 4 \mathrm{~cm}$ space group of $\mathrm{MAPbI}_{3}$ found in the literature ${ }^{27,28}$. To form a freestanding stable wafer, the powder was compacted for $30 \mathrm{~min}$ at room temperature using a hydraulic press with a pressure of $75.5 \mathrm{MPa}$. The compactness of these wafers with a resulting thickness of $230 \mu \mathrm{m}$ is $88 \%$ of the theoretical limit calculated from the simulated lattice parameters ${ }^{27,28}$ and is in good agreement with the density versus pressure curve shown in Supplementary Fig. 2 (generated as the calibration plot).

The second manufacturing phase is shown in Supplementary Fig. 4. First, a photoresist grid with $10 \mu \mathrm{m}$ height is build up on the backplane by photolithography. A top view of the regular grid structure with a pixel pitch of $50 \mu \mathrm{m}$ and fill factor of $58 \%$ is shown in the scanning electron microscopy (SEM) image in Fig. 2a. The total active area of the X-ray detector is $3.2 \times 2.4 \mathrm{~cm}^{2}$. Figure $2 \mathrm{~b}$ shows a micrograph of the grid structure, in which the bottom electrode array can be seen as bright areas. In the second step, the grid on the backplane is filled with $\mathrm{MAPbI}_{3}$ powder and then liquefied under a methylamine atmosphere. More details on this reaction can be found elsewhere ${ }^{29-31}$. In the timescale of seconds, the previously prepared $\mathrm{MAPbI}_{3}$ wafer is placed on the liquid phase. Fixation occurs after the liquefied $\mathrm{MAPbI}_{3}$ recrystallizes due to the evaporation of excessive methylamine during the annealing step at $50^{\circ} \mathrm{C}$, acting as an adhesion promoter between the wafer and backplane, as shown in Fig. 2c.

To investigate the recrystallized grains, SEM cross-section images were obtained, as shown in Fig. $2 \mathrm{~d}-\mathrm{f}$. During the bonding process, the liquification of the $\mathrm{MAPbI}_{3}$ wafer due to methylamine vapours is not excluded, but it will be very limited since the thickness of the recrystallized layer slightly exceeds the grid height. The bottom part of the $\mathrm{MAPbI}_{3}$ wafer bonded to an indium tin oxide glass with grid structures at the glass interface is shown in Fig. $2 \mathrm{~d}$. The surface of the cross section is rough because of the breaking mechanism of the wafer and glass. Morphology differences between the recrystallized and soft-sintered perovskite can be identified. A closer look inside the soft-sintered absorber is shown in Fig. 2e. The grain size varies between 0.5 and $5.0 \mu \mathrm{m}$ and some pores are visible between the powder grains, which are attributed to mechanical soft sintering. In contrast, recrystallized $\mathrm{MAPbI}_{3}$ has fewer pores between the grains (Fig. 2f), which has a smaller size of up to $2 \mu \mathrm{m}$. The sensitivity and thus the charge transport properties of methylamine-treated $\mathrm{MAPbI}_{3}$ wafer show no notable change compared with a non-treated one. Nevertheless, additional defect states may have been introduced by changing the grain size $\mathrm{s}^{32-34}$.

A great advantage of the presented manufacturing process of the X-ray imager is the possibility to execute quality control of the $\mathrm{MAPbI}_{3}$ wafer before attachment to the backplane. In fact, freestanding wafers can be X-rayed themselves, and the homogeneity of $\mathrm{X}$-ray absorption can be controlled using a commercially available flat-panel detector (Supplementary Fig. 5a-c).

\section{Characterization of freestanding wafers}

For a better understanding of the imaging X-ray detector properties, a closer look at the compact wafer is required. Therefore, we have performed impedance measurements on a single device made of a soft-sintered $\mathrm{MAPbI}_{3}$ wafer with platinum $(\mathrm{Pt})$ and $\mathrm{Cr}$ electrodes (Supplementary Fig. 6a,b). A schematic of the wafer stack is shown in Supplementary Fig. 11a. According to the resulting geometrical capacitance and sample dimensions, the dielectric constant of the wafer is $\varepsilon=75$. This is in good agreement with previously reported values $^{35-37}$. For dark current measurements, a $977-\mu \mathrm{m}$-thick, $86 \%$ dense wafer has been used.

The Cr electrode was grounded during the measurements, and the applied voltage was in the range of -200 to $+200 \mathrm{~V}$, corresponding 
a

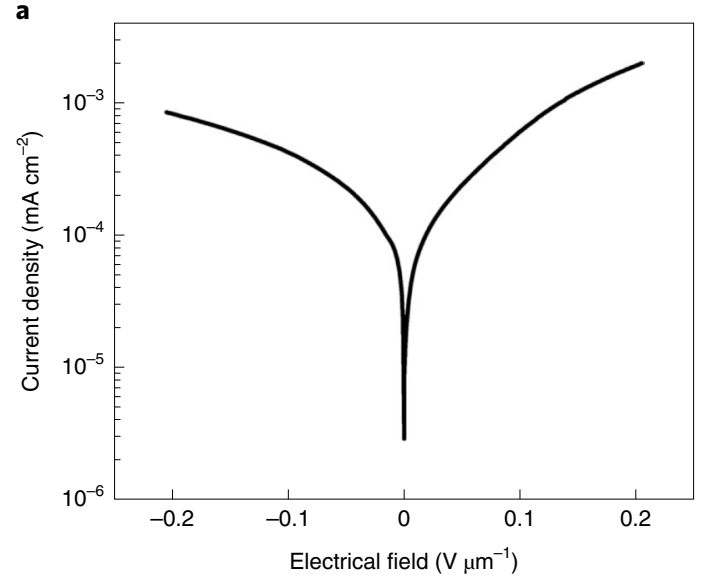

c

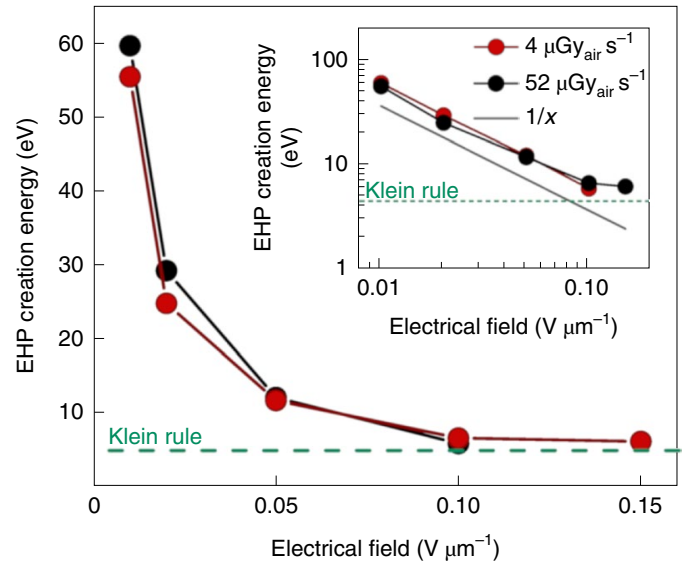

b

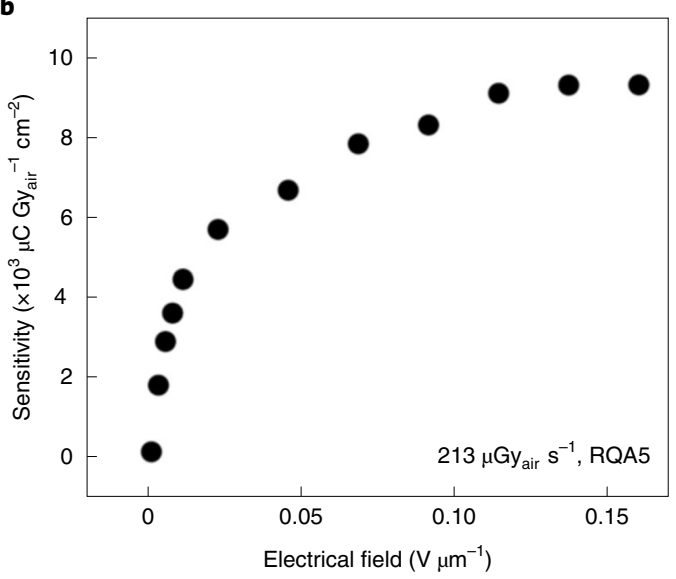

d

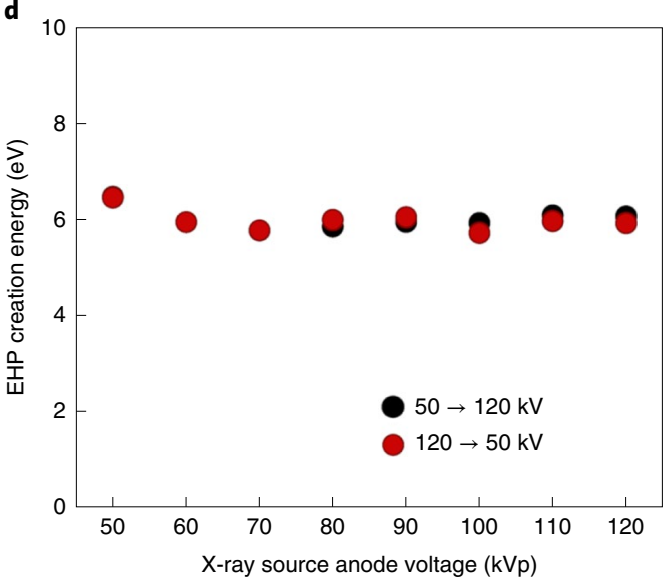

Fig. 3 | Optoelectronic properties of $\mathrm{MAPbl}_{3}$ wafers. $\mathbf{a}$, Current density measurement under dark condition for an $\mathrm{MAPbl}_{3}$ wafer. The applied voltage ranges from -200 to $+200 \mathrm{~V}$, which corresponds to an electrical field of $\pm 0.2 \mathrm{~V} \mu \mathrm{m}^{-1}$. b. Calculated sensitivity (S) of the X-ray responses in the RQA5 spectrum and a dose of $213 \mu \mathrm{Gy}_{\text {air }} \mathrm{s}^{-1}$. c, EHP creation energy for the measurement series of 52 and $4 \mu \mathrm{Gy}_{\text {air }} \mathrm{s}^{-1}$. The limit is $4.5 \mathrm{eV}$ according to the Klein rule $^{40}$. In the double logarithmic representation (inset), the hyperbolic behaviour of $W_{ \pm}$is visible. d, EHP creation energy versus exposed X-ray spectrum. The $\mathrm{MAPbl}_{3}$ wafer was exposed with a dose of $40 \mu \mathrm{Gy}_{\text {air }}$ in the RQA5 spectrum and an applied electrical field of $0.043 \mathrm{~V} \mu \mathrm{m}^{-1}$. The $\mathrm{X}$-ray tube voltage was varied from 50 to $120 \mathrm{kVp}$ (black points) and back to $50 \mathrm{kVp}$ thereafter (red dots).

to an electrical field of $\pm 0.2 \mathrm{~V} \mu \mathrm{m}^{-1}$. The electrical field was at

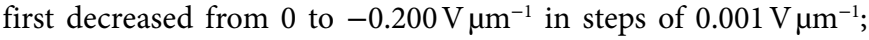

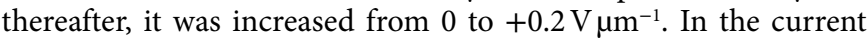
density versus electrical field $(J-E)$ plot (Fig. 3a), the dark current density reaches the maximum value of $8.40 \times 10^{-4} \mathrm{~mA} \mathrm{~cm}^{-2}$ for negative fields, the so-called reverse bias direction, and $1.98 \times 10^{-3} \mathrm{~mA} \mathrm{~cm}^{-2}$ for positive (forward) bias. This leads to a dark resistivity of $\sim 2.4 \times 10^{9} \Omega \mathrm{cm}$, which is in the same order of magnitude to the values reported in ref. ${ }^{38}$. This small rectifying behaviour could be caused by the used electrodes and their different work functions of $\phi_{\mathrm{m}, \mathrm{Cr}}=4.5 \mathrm{eV}$ and $\phi_{\mathrm{m}, \mathrm{Pt}}=5.7 \mathrm{eV}\left(\right.$ ref. $\left.^{39}\right)$ or ions and their associated vacancies ${ }^{32}$.

The response of the wafer to 2-s-long irradiation with the RQA5 (according to the IEC 61267:2005 standard) X-ray spectrum and a dose rate of $213 \mu \mathrm{Gy}_{\text {air }} \mathrm{s}^{-1}$ at different bias voltages was captured with a Keithley 2400 source meter. This results in the maximum value of $9,300 \mu \mathrm{CGy}_{\text {air }}{ }^{-1} \mathrm{~cm}^{-2}$ for the sensitivity of our wafer at an electrical

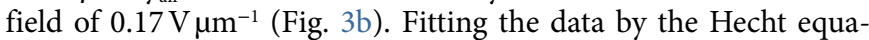
tion $^{10}$ (red curve in Supplementary Fig. 11b) results in a $\mu \tau$ product of approximately $4 \times 10^{-4} \mathrm{~cm}^{2} \mathrm{~V}^{-1}$. A comparison with previously reported perovskite materials shows that the measured sensitivity is in good agreement with printed $\mathrm{MAPbI}_{3}$ samples $^{17}$, 3.5 times higher than the soft-sintered $\mathrm{MAPbI}_{3}$ wafers ${ }^{18}$ and over 100 times better than single-crystal $\mathrm{MAPbBr}_{3}\left(\right.$ ref. $\left.{ }^{16}\right)$.
Another important figure of merit in comparing X-ray detectors with different thicknesses and materials is the electron-hole pair (EHP) generation energy $W_{ \pm}$. $W_{ \pm}$is the amount of absorbed radiation energy needed to create a single free EHP (Supplementary Information provides more information $)^{40,41}$. In Fig. 3c, the values of $W_{ \pm}$are plotted as a function of the applied bias for two dose rates. $W_{ \pm}$decreases with an approximately hyperbolic $(f(x)=1 / x)$ behaviour with increasing electrical field and approaches the empirical limit of $W_{ \pm}=3 E_{\mathrm{G}} \approx 4.5 \mathrm{eV}$ ( $E_{\mathrm{G}}$ is the bandgap), which is known as the Klein rule ${ }^{40}$. $W_{+}$values (on a linear scale) for a total of six different dose rates are shown in Supplementary Fig. 12a. Within the measurement accuracy, $W_{ \pm}$is independent of the dose rate for electrical fields higher than $0.05 \mathrm{~V} \mu \mathrm{m}^{-1}$, indicating an almost full extraction of the generated charges.

To investigate the dependencies of $W_{ \pm}$with the X-ray photon energy, we performed a series of X-ray response measurements with different photon spectra obtained by varying the anode voltages on the X-ray tube. The simulated X-ray photon spectra are shown in Supplementary Fig. 12b. With the help of these photon energy densities, the absorbed energy for the $\mathrm{MAPbI}_{3}$ wafer can be determined: for an anode voltage of $50 \mathrm{kVp}$, the wafer absorbed $98.63 \%$ of the emitted X-ray spectrum. The absorbed energy decreases to $71.33 \%$ for increasing anode voltages up to $120 \mathrm{kVp}$ (Supplementary Table 1). The wafer was irradiated for $1 \mathrm{~s}$ each time with a dose of $40 \mu \mathrm{Gy}_{\text {air }}$ under an 
a
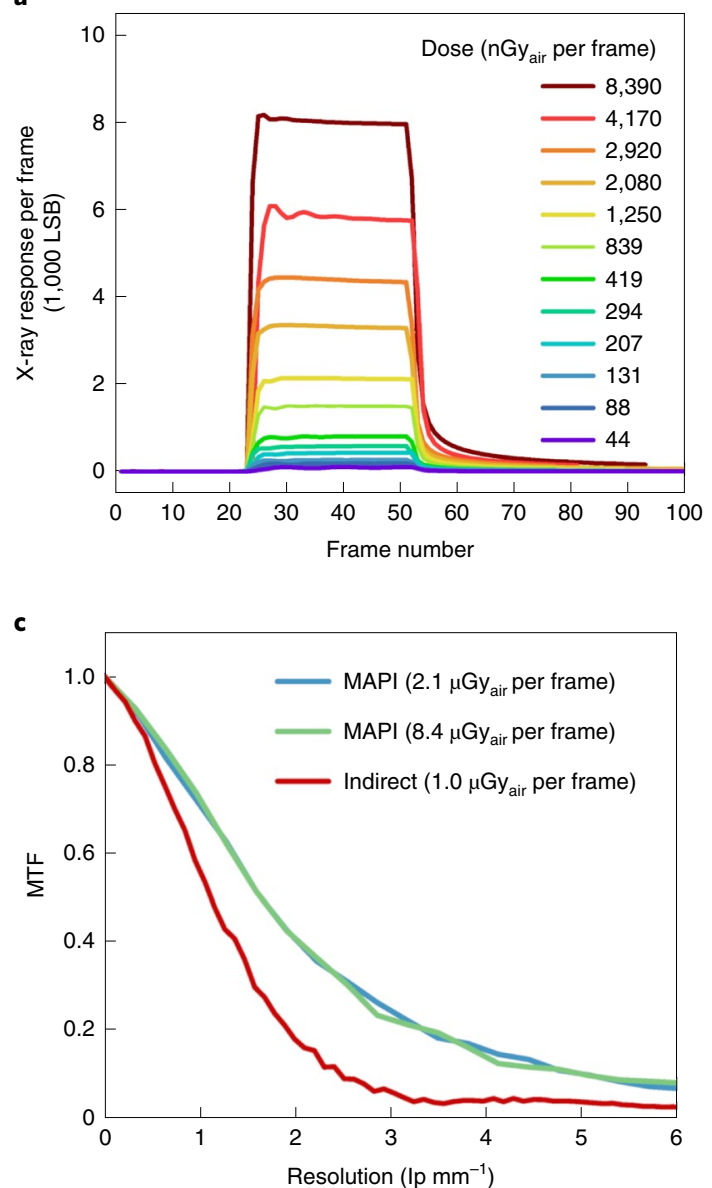

e

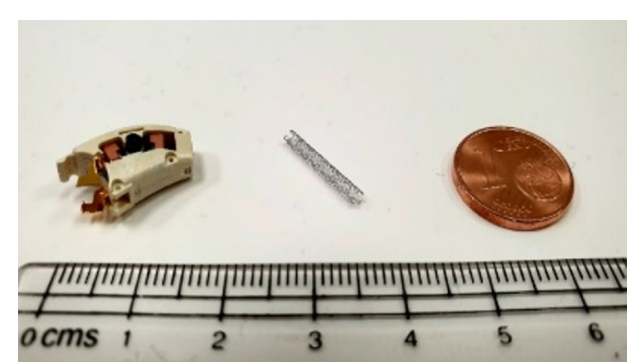

b

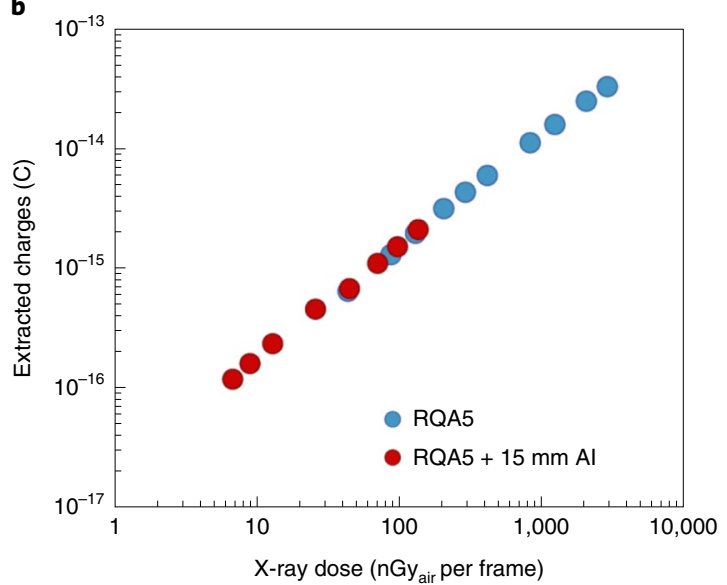

d
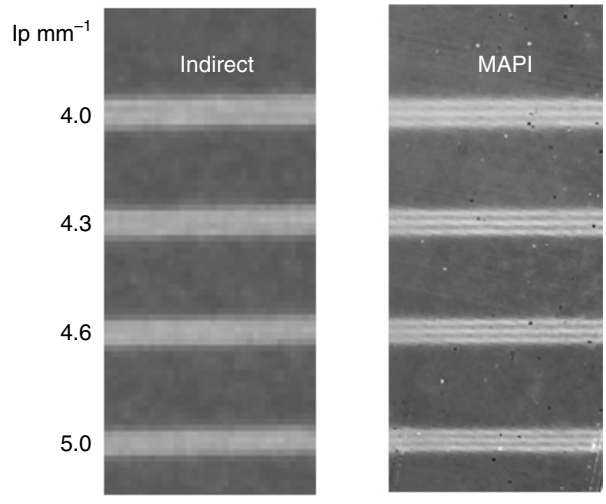

f
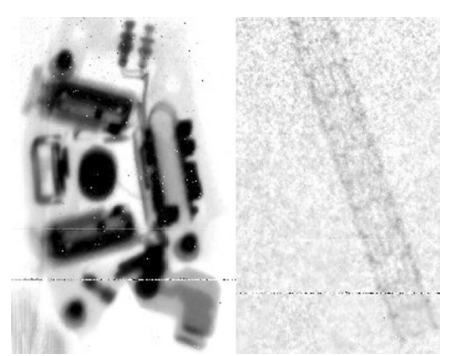

Fig. 4 | X-ray imaging properties of $\mathbf{M A P b l}_{3}$ detector at RQA5. $\mathbf{a}, \mathrm{X}$-ray response of the pixelated $\mathrm{MAPbl}_{3}$ imager under an electrical field of $0.03 \mathrm{~V} \mu \mathrm{m}^{-1}$ at different doses. b, Linearity plot of the extracted charges versus dose in the RQA5 (blue) and 15 mm Al-filtered RQA5 (red) spectrum. c, MTF curves of the pixelated $\mathrm{MAPbl}_{3}$ imager for two different doses (blue and green) and MTF of commercial indirect-conversion detector based on the complementary metal-oxide-semiconductor technology (red). $\mathbf{d}, \mathrm{X}$-ray image with the resolution of phantom structures taken by the indirect-conversion (left) and $\mathrm{MAPbl}_{3}$ detector (MAPI; right). e, Photograph of part of a hearing aid (left) and a metallic coronary stent (right). $\mathbf{f}$, X-ray image of the hearing aid (left) and coronary stent (right) taken by the pixelated $\mathrm{MAPbl}_{3}$ detector at a dose of $4.17 \mu \mathrm{Gy}_{\text {air }}$ per frame. Even the mesh of the stent with a cross-section size of $100 \mu \mathrm{m} \times 200 \mu \mathrm{m}$ can be resolved.

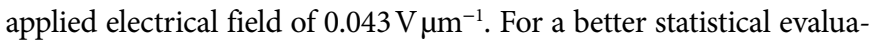
tion and excluding possible degradation factors, the anode voltage was initially increased from 50 to $120 \mathrm{kVp}$ and then decreased from 120 to $50 \mathrm{kVp}$. The corresponding X-ray pulses show an increasing pulse height with higher anode voltages with the maximum value at around $100 \mathrm{kVp}$ (Supplementary Fig. 12c). The increase is proportional to the photon energy fluence of the X-ray tube. The resulting average $W_{ \pm}$ values are $5.99 \pm 0.20 \mathrm{eV}$ (Fig. $3 \mathrm{~d}$ ), that is, $W_{ \pm}$is independent of the $\mathrm{X}$-ray photon energy. This is of great importance since $\mathrm{X}$-ray photons with a lower energy carry higher diagnostic information.

Furthermore, no degradation caused by irradiation has been observed after a cumulative dose of $11 \mathrm{~Gy}_{\text {air }}$ (Supplementary Fig.
13). Similar to $\mathrm{MAPbI}_{3}$, the theoretical limit of $W_{ \pm}$in amorphous selenium (a-Se) ranges between 5 and $6 \mathrm{eV}$. In practice, however, values of $40-50 \mathrm{eV}$ at an electrical field of $10 \mathrm{~V} \mathrm{\mu m}^{-1}$ are achieved ${ }^{42}$. In addition, a-Se shows decreasing $W_{ \pm}$for increasing $\mathrm{X}$-ray photon energy ${ }^{42}$. All the results presented here with $\mathrm{MAPbI}_{3}$ wafer devices show the great potential of this material class for X-ray detector application.

\section{Characterization of X-ray detectors}

With the knowledge of freestanding wafer measurements, we were able to manufacture a pixelated $\mathrm{MAPbI}_{3} \mathrm{X}$-ray imaging detector with outstanding performance. X-ray characterization was performed by 
varying the dose range over five orders of magnitude initially using the RQA5 spectrum and adding different filters later. For the corresponding photon energy densities, see Supplementary Fig. 14a. The level of absorption of these three spectra for different thicknesses of $\mathrm{MAPbI}_{3}$ is shown in Supplementary Fig. 14b. With the effective thickness of our wafer (red dashed line), we obtained theoretical absorption values of $50 \%, 46 \%$ and $36 \%$ for the RQA5, RQA5 with $15 \mathrm{~mm} \mathrm{Al}$ filter and RQA $5+1 \mathrm{~mm} \mathrm{~Pb}$ spectra, respectively.

For the investigation of the imager response to 1-s-long X-ray pulses, video sequences were taken with an integration time of $35 \mathrm{~ms}$ per frame. Frame sequences were acquired in the rolling shutter mode without synchronization between the X-ray source and $\mathrm{X}$-ray detector. The offset-corrected signals under the maximum

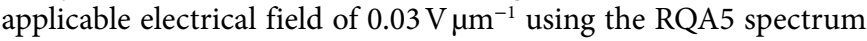
with doses ranging from 44 to $8,390 \mathrm{nGy}_{\text {air }}$ per frame are presented in Fig. 4a. The pulses show good time response, as indicated by the steep increase and decrease. To evaluate the linearity of the imager responses for a wider dose range, we used the RQA5 spectrum in combination with additional $15 \mathrm{~mm}$ Al filtration. This detector is impressive, with good linearity over a dose range of three orders of magnitude (Fig. 4b and Supplementary Fig. 15a). Supplementary Fig. 15b shows a close-to-ideal dose dependence (slope, 0.99).

Image lag describes the amount of charge that was carried over from the previous to the next image frame ${ }^{21}$. In Supplementary Fig. 15c, image lags for different doses after 1 frame ( $35 \mathrm{~ms}), 5$ frames $(175 \mathrm{~ms})$ and 10 frames $(350 \mathrm{~ms})$ are shown. In comparison to a-Se and amorphous silicon, $\mathrm{MAPbI}_{3}$ shows higher lag: lags of around $1.2 \%$ for amorphous silicon and $0.7 \%$ for a-Se are detected after $330 \mathrm{~ms}$ (refs. ${ }^{43,44}$ ). The image lag of $\mathrm{MAPbI}_{3}$ after 1 frame is similar to polycrystalline CZT and better for higher frames ${ }^{14}$. Since the applied electrical field $\left(0.03 \mathrm{~V} \mathrm{\mu m}^{-1}\right)$ on our detector is rather low, we expect a much lower image lag for higher electric fields. The sensitivity varies between 1,010 and $1,060 \mu \mathrm{CGy}_{\text {air }}{ }^{-1} \mathrm{~cm}^{-2}$ (Supplementary Fig. 16a). A comparison with previously reported detector materials shows that the measured sensitivity is four times higher than CZT (refs. ${ }^{12,13}$ ) and up to 60 times better than a-Se (ref. ${ }^{10}$ ).

Another key parameter for the imager performance is the modulation transfer function (MTF), that is, the spatial resolution or relative response as a function of the spatial frequency ${ }^{44}$. The MTF plot (Fig. 4c) shows that for the measurement range used here, the MTF is independent of the X-ray dose. For comparing with another $\mathrm{X}$-ray imaging detector mostly used for radiography application, an indirect-conversion complementary metal-oxide-semiconductor detector (Xineos-2222HS, Teledyne DALSA) was chosen. The associated MTF (Fig. 4c, red curve) is obviously worse than that of the $\mathrm{MAPbI}_{3}$ detector. A further comparison of the two detectors is shown in Fig. $4 \mathrm{~d}$. The image made by the indirect-conversion detector is blurred, and none of the resolution phantoms can be resolved. In contrast, $5.0 \mathrm{lp} \mathrm{mm}^{-1}$ can be resolved using our $\mathrm{MAPbI}_{3}$ detector.

To prove the good resolution, part of a hearing aid and a coronary stent (diameter, $2 \mathrm{~mm}$; length, $15 \mathrm{~mm}$; rectangular mesh cross section, $100 \mu \mathrm{m}$ x $200 \mu \mathrm{m}$ (Supplementary Fig. 17)) were X-rayed with an exposure of $4.17 \mu \mathrm{Gy}_{\text {air }}$ per frame in the RQA5 spectrum. The photograph of the objects is shown in Fig. 4e. The resulting $\mathrm{X}$-ray images offer a detailed view of the structures examined; even the mesh of the stent (Fig. 4f, right) is clearly visible. These results show the outstanding potential of the $\mathrm{MAPbI}_{3}$ imaging detector fabricated by our two-step manufacturing process for use in the medical field.

\section{Conclusions}

We have reported a two-step manufacturing process for $\mathrm{MAPbI}_{3}$ $\mathrm{X}$-ray flat-panel detectors based on the mechanical sintering of a freestanding absorber layer and integration of this layer on a pixelated backplane. A photoresist grid functions as a mechanical anchor and recrystallized $\mathrm{MAPbI}_{3}$ acts as the adhesion promoter for the soft-sintered thick $\mathrm{MAPbI}_{3}$ absorber layer. We used our approach to create a pixelated X-ray detector with a resolution of

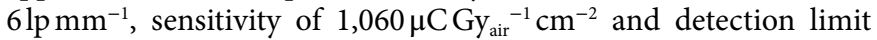
of $0.22 \mathrm{nGy}_{\text {air }}$ per frame at an applied electrical field of $0.03 \mathrm{~V} \mathrm{\mu m}^{-1}$. The EHP creation energy $W_{ \pm}$of $12.4 \mathrm{eV}$ is still higher than the empirical limit of $4.5 \mathrm{eV}$ given by the Klein rule ${ }^{40}$, but we have shown-via measurements on freestanding wafers-that a $W_{ \pm}$ value of $5.99 \mathrm{eV}$ should be achievable when applying an electrical

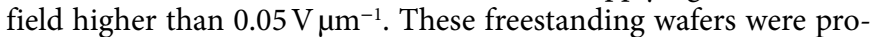
duced with the same soft-sintering approach as the X-ray detector and confirmed the electrical transport properties of $\mathrm{MAPbI}_{3}$. A value of $9,300 \mu \mathrm{CGy}_{\text {air }}{ }^{-1} \mathrm{~cm}^{-2}$ could be achieved for sensitivity and $4 \times 10^{-4} \mathrm{~cm}^{2} \mathrm{~V}^{-1}$ for the $\mu \tau$ product. We also showed that the $W_{ \pm}$ value of our freestanding $\mathrm{MAPbI}_{3}$ devices is independent of the $\mathrm{X}$-ray photon energy. Hence, the freestanding compact $\mathrm{MAPbI}_{3}$ wafer has a very high potential for stable and excellent detection over the whole energy range of X-ray applications.

We have illustrated that this technology can be scaled to large detection areas via integration on a 508 pixels per inch backplane with $640 \times 480$ pixels. To further improve the performance (sensitivity and dynamic behaviour) of the pixelated $\mathrm{MAPbI}_{3}$ detector to the level shown for freestanding wafers, the backplane must be tailored to accommodate a higher electric field. Additional interlayer engineering of the detector stack is required to further reduce the dark current. The development of such X-ray detectors with high resolution and sensitivity can-we believe-speed up the translation of AI to routine clinical practice in X-ray imaging applications and help improve healthcare outcomes. In the short term, applications in the field of general radiography, fluoroscopy, angiography and neurology could benefit from improved resolution, which today is an option only in mammography. Moreover, mammography will benefit from improved sensitivities, which are typical for indirect-conversion detectors. Improved sensitivities can also lead to lower X-ray doses but with similar or improved image quality. Currently, these different applications rely on different technologies-our $\mathrm{MAPbI}_{3} \mathrm{X}$-ray imaging detector could potentially provide a single technology for all of them.

\section{Methods}

Device manufacturing. For this paper, two different devices were used. For the first device, freestanding $\mathrm{CH}_{3} \mathrm{NH}_{3} \mathrm{PbI}_{3}\left(\mathrm{MAPbI}_{3}\right)$ wafers with a diameter of $15 \mathrm{~mm}$ and thickness between 880 and $1,000 \mu \mathrm{m}$ have been produced using commercially available $\mathrm{MAPbI}_{3}$ powder (Xi'an Polymer Light Technology). The powder was sieved with a $50 \mu \mathrm{m}$ mesh. The powder was filled into a height-adjustable powder container and a cylinder of stainless steel with a polished surface was placed above this. The hydraulic press (PerkinElmer) can apply up to $9 \mathrm{t}$, which corresponds to a pressure of $495 \mathrm{MPa}$ for the wafers. The soft sintering of the wafers was done at a pressure of $110 \mathrm{MPa}(2 \mathrm{t})$, applied for $30 \mathrm{~min}$ at a temperature of $70^{\circ} \mathrm{C}$. On the two-wafer surfaces, electrodes with an area of $1 \mathrm{~cm}^{2}$ were deposited via physical vapour deposition. For that purpose, $100 \mathrm{~nm} \mathrm{Cr}$ on one side and $100 \mathrm{~nm}$ Pt on the other side were sputtered.

The second device is the X-ray image sensor having the following parts. The backplane consists of a indium gallium zinc oxide thin-film transistor (TFT) array comprising $640 \times 480$ pixels with a pixel pitch of $50 \mu \mathrm{m}$ and a resolution of 508 pixels per inch. The negative photoresist grid, with a height of $10 \mu \mathrm{m}$, comprised a mixture of SU-8 50 (Kayaku Advanced Materials and distributed by micro resist technology) and cyclopentanone (Sigma-Aldrich) (5:1); it was deposited on the backplane by spin coating at 3,000 rounds per minute and structured by optical lithography. $\mathrm{MAPbI}_{3}$ powder was filled into the grid-like structure and liquefied under a methylamine gas (Sigma-Aldrich) atmosphere. Fabrication of the absorber layer was performed using a slightly modified procedure presented in ref. ${ }^{1}$. The wafer was pressed at $9 \mathrm{t}(75.5 \mathrm{MPa})$ and at room temperature for $30 \mathrm{~min}$. On top of this wafer, an 80 -nm-thick $\mathrm{Cr}$ electrode and $100 \mathrm{~nm}$ gold $\mathrm{(Au})$ were sputtered. The manufactured wafer was placed on the liquid $\mathrm{MAPbI}_{3}$ and fixed after its recrystallization. To ensure good evaporation of excess methylamine gas, the sample was placed on a heating plate at $50^{\circ} \mathrm{C}$ for $30 \mathrm{~min}$. The stacked layers were encapsulated by laminating a high barrier film (TESA 61572) to avoid degradation due to external stimuli.

Image readout and processing for pixelated $\mathrm{X}$-ray detector. Images from the $480 \times 640$ pixels TFT panel were read by a commercially available ROIC (AD71124, 
Analog Devices). The signal at the input was simultaneously integrated, amplified, low-pass filtered and converted from analogue to digital with a 16-bit converter. The integrator feedback capacitance $C_{\mathrm{f}}$ was $0.125 \mathrm{pF}$ and the integration time was $35 \mathrm{~ms}$. The readout occurred by the rolling shutter principle, and the X-ray tube and detector were not synchronized. The acquiring frame rate was $\sim 28.6$ frames per second. To eliminate fixed pattern noise (offset compensation), an offset map was generated by averaging 100 dark images, which was subtracted from the images. The mean value and standard deviation were deduced from the ROI (Supplementary Fig. 18). The standard deviation in the ROI represents the electronic noise in LSB ( 11 LSB). Noise in the image sensor in terms of electrons (considering 48 electrons per LSB) is 535 electrons. X-ray recordings from objects were offset and compensated by subtracting the offset map and flat-field corrected by calculating the gain factor of each pixel for a flat-field image. The MTF is determined by the slanted-edge method ${ }^{45}$. First, an object with a sharp tungsten edge is placed on the X-ray detector and the edge profile is derived from the resulting X-ray image. The line-spread function is derived by differentiating the edge profile. The Fourier transform of the line-spread function defines the MTF.

Electrical characterization of freestanding wafers. The current density measurement was performed with a Keithley 2400 source meter sampling at $10 \mathrm{~Hz}$ that was connected to the sample holder filled with argon gas. The MAPbI ${ }_{3}$ wafers are measured in an inert atmosphere and therefore protected from moisture and light. For measurement during X-ray exposure, the sample holder is placed in an Al box underneath a MEGALIX Cat Plus 125/40/90 (Siemens Healthineers AG) $\mathrm{X}$-ray source with a tungsten anode. The distance between the sample and X-ray source is $120 \mathrm{~cm}$. The X-ray dose was varied by changing the tube current by over two orders of magnitude, measured with a PTW Diados T11003-001896 dosimeter and adjusted with correction factors provided in its datasheet for anode voltages other than $70 \mathrm{kVp}$. For more details, see Supplementary Information.

SEM. To obtain high-resolution SEM images of the $\mathrm{MAPbI}_{3}$ perovskite layers, a Schottky field-emission SEM (JEOL JSM-7610F) was used at an acceleration voltage of $15 \mathrm{kV}$. For the cross-sectional image, the glass of the imager was scratched with a diamond pen and then broken by hand. SEM images of the grid photoresist were obtained by an FEI Quanta 3D FEG microscope, using a $5 \mathrm{kV}$ electron beam and a secondary electron detector.

$\mathrm{X}$-ray diffraction measurement. The structural analysis of the $\mathrm{MAPbI}_{3}$ powder consists of XRD measurements performed by classical ex situ Bragg-Brentano geometry using a Panalytical X'pert powder diffractometer with filtered $\mathrm{Cu} \mathrm{K} \alpha$ radiation and an X'Celerator solid-state strip detector.

\section{Data availability}

The datasets analysed in this study are available from the corresponding authors upon reasonable request.

Received: 23 March 2021; Accepted: 12 August 2021; Published online: 23 September 2021

\section{References}

1. LeCun, Y., Bengio, Y. \& Hinton, G. Deep learning. Nature 521, 436-444 (2015).

2. Hosny, A. et al. Artificial intelligence in radiology. Nat. Rev. Cancer 18, 500-510 (2018)

3. Topol, E. J. High-performance medicine: the convergence of human and artificial intelligence. Nat. Med. 25, 44-56 (2019).

4. Grace, K. et al. When will AI exceed human performance? Evidence from AI experts. J. Artif. Intell. Res. 62, 729-754 (2018).

5. Sabottke, C. F. \& Spieler, B. M. The effect of image resolution on deep learning in radiography. Radiol. Artif. Intell. 2, e190015 (2020).

6. Moy, J.-P. Recent developments in X-ray imaging detectors. Nucl. Instrum. Meth. A 442, 26-37 (2000).

7. Chotas, H. G., Dobbins, J. T. III \& Ravin, C. E. Principles of digital radiography with large-area, electronically readable detectors: a review of the basics. Radiology 210, 595-599 (1999).

8. Holland, S. E., Wang, N. W. \& Moses, W. W. Development of low noise, back-side illuminated silicon photodiode arrays. IEEE Trans. Nucl. Sci. 44 443-447 (1997).

9. Stoumpos, C. C. et al. Crystal growth of the perovskite semiconductor $\mathrm{CsPbBr}$ : a new material for high-energy radiation detection. Cryst. Growth Des. 13, 2722-2727 (2013).

10. Kasap, S. O. X-ray sensitivity of photoconductors: application to stabilized a-Se. J. Phys. D 33, 2853 (2000).

11. Hunter, D. M., Belev, G., Kasap, S. O. \& Yaffe, M. J. Measured and calculated $K$-fluorescence effects on the MTF of an amorphous-selenium based CCD X-ray detector. Med. Phys. 39, 608-622 (2012).

12. Ivanov, Yu,M. et al. The possibilities of using semi-insulating CdTe crystals as detecting material for X-ray imaging radiography. Phys. Stat. Sol. C 3, 840-844 (2003).
13. Bellazzini, R. et al. Chromatic X-ray imaging with a fine pitch CdTe sensor coupled to a large area photon counting pixel ASIC. J. Instrum. 8, C02028 (2013).

14. Tokuda, S. et al. Improvement of the temporal response and output uniformity of polycrystalline CdZnTe films for high-sensitivity X-ray imaging. Proc. SPIE 5030, 861-870 (2003).

15. Yakunin, S. et al. Detection of X-ray photons by solution-processed lead halide perovskites. Nat. Photon. 9, 444-449 (2015).

16. Wei, H. et al. Sensitive X-ray detectors made of methylammonium lead tribromide perovskite single crystals. Nat. Photon. 10, 333-339 (2016).

17. Kim, Y. C. et al. Printable organometallic perovskite enables large-area, low-dose X-ray imaging. Nature 550, 87-91 (2017).

18. Shrestha, S. et al. High-performance direct conversion X-ray detectors based on sintered hybrid lead triiodide perovskite wafers. Nat. Photon. 11, 436-440 (2017).

19. Ye, Fei et al. High-quality cuboid $\mathrm{CH}_{3} \mathrm{NH}_{3} \mathrm{PbI}_{3}$ single crystals for high performance X-ray and photon detectors. Adv. Funct. Mater. 29, 1806984 (2019).

20. Huang, Yanmin et al. A-site cation engineering for highly efficient $\mathrm{MAPbI}_{3}$ single-crystal X-ray detector. Angew. Chem. Int. Ed. 58, 17834-17842 (2019).

21. Kabir, M. Z. \& Kasap S. O. in Springer Handbook of Electronic and Photonic Materials (eds Kasap, S. O. \& Capper, P.) https://doi.org/10.1007/978-3-31948933-9_45 (Springer, 2017).

22. Zhao, J. et al. Perovskite-filled membranes for flexible and large-area direct-conversion X-ray detector arrays. Nat. Photon. 14, 612-617 (2020).

23. Kronemeijer, A. J. et al. P-127: dual-gate self-aligned IGZO TFTs monolithically integrated with high-temperature bottom moisture barrier for flexible AMOLED. SID Symp. Dig. Tec. 49, 1577-1580 (2018).

24. Tordera, D. et al. A high-resolution thin-film fingerprint sensor using a printed organic photodetector. Adv. Mater. Technol. 4, 1900651 (2019).

25. Shrivastava, A. \& Gupta, V. B. Methods for the determination of limit of detection and limit of quantitation of the analytical methods. Chron. Young Sci. 2, 21-25 (2011).

26. Wei, Haotong et al. Dopant compensation in alloyed $\mathrm{CH}_{3} \mathrm{NH}_{3} \mathrm{PbBr}_{3-x} \mathrm{Cl}_{x}$ perovskite single crystals for gamma-ray spectroscopy. Nat. Mater. 16, 826-833 (2017).

27. Guo, X. et al. Identification and characterization of the intermediate phase in hybrid organic-inorganic $\mathrm{MAPbI}_{3}$ perovskite. Dalton Trans. 45, 3806-3813 (2016).

28. Stoumpos, C. C., Malliakas, C. D. \& Kanatzidis, M. G. Semiconducting tin and lead iodide perovskites with organic cations: phase transitions, high mobilities, and near-infrared photoluminescent properties. Inorg. Chem. 52, 9019-9038 (2013).

29. Zhou, Z. et al. Methylamine-gas-induced defect-healing behavior of $\mathrm{CH}_{3} \mathrm{NH}_{3} \mathrm{PbI}_{3}$ thin films for perovskite solar cells. Angew. Chem. 127, 9841-9845 (2015)

30. Jiang, Y. et al. Post-annealing of $\mathrm{MAPbI}_{3}$ perovskite films with methylamine for efficient perovskite solar cells. Mater. Horiz. 3, 548-555 (2016).

31. Jacobs, D. L. \& Zang, L. Thermally induced recrystallization of $\mathrm{MAPbI}_{3}$ perovskite under methylamine atmosphere: an approach to fabricating large uniform crystalline grains. Chem. Commun. 52, 10743-10746 (2016).

32. Sherkar, T. S. et al. Recombination in perovskite solar cells: significance of grain boundaries, interface traps, and defect ions. ACS Energy Lett. 2, 1214-1222 (2015).

33. Son, D.-Y. et al. Self-formed grain boundary healing layer for highly efficient $\mathrm{CH}_{3} \mathrm{NH}_{3} \mathrm{PbI}_{3}$ perovskite solar cells. Nat. Energy 1, 16081 (2016).

34. Park, J.-S. et al. Accumulation of deep traps at grain boundaries in halide perovskites. ACS Energy Lett. 4, 1321-1327 (2019).

35. Onoda-Yamamuro, N., Matsuo, T. \& Suga, H. Dielectric study of $\mathrm{CH}_{3} \mathrm{NH}_{3} \mathrm{~Pb} X_{3}(X=\mathrm{Cl}, \mathrm{Br}, \mathrm{I})$. J. Phys. Chem. Solids 53, 935-939 (1992).

36. Govinda, S. et al. Behavior of methylammonium dipoles in $\mathrm{MAPb}_{3}(X=\mathrm{Br}$ and I). J. Phys. Chem. Lett. 8, 4113-4121 (2017). 8.

37. Wilson, J. N. et al. Dielectric and ferroic properties of metal halide perovskites. APL Mater. 7, 010901 (2019).

38. Garcia-Battle, M. et al. Mobile ion-driven modulation of electronic conductivity explains long-timescale electrical response in lead iodide perovskite thick pellets. ACS Appl. Mater. Interfaces 13, 35617-35624 (2021)

39. Skriver, H. L. \& Rosengaard, N. M. Surface energy and work function of elemental metals. Phys. Rev. B 46, 7157 (1992).

40. Klein, C. A. Bandgap dependence and related features of radiation ionization energies in semiconductors. J. Appl. Phys. 39, 2029-2038 (1968).

41. Que, W. \& Rowlands, J. A. X-ray photogeneration in amorphous selenium: geminate versus columnar recombination. Phys. Rev. B 51, 10500 (1995).

42. Kabir, M. Z., Arnab, S. M. \& Hijazi, N. Electron-hole pair creation energy in amorphous selenium: geminate versus columnar recombination. J. Mater. Sci. Mater. Electron. 30, 21059-21063 (2019).

43. Granfors, P. R. et al. Performance of a $41 \times 41 \mathrm{~cm}^{2}$ amorphous silicon flat panel X-ray detector designed for angiographic and R\&F imaging applications. Med. Phys. 30, 2715-2726 (2003). 
44. Choquette, M. et al. Performance of a real-time selenium-based X-ray detector for fluoroscopy. Proc. SPIE 4320, 501-508 (2001).

45. Samei, E., Flynn, M. J. \& Reimann, D. A. A method for measuring the presampled MTF of digital radiographic systems using an edge test device. Med. Phys. 25, 102-113 (1998).

\section{Acknowledgements}

This work has received funding from the European Union's Horizon 2020 research and innovation programme under the Photonics Public Private Partnership (www. photonics21.org) with project PEROXIS under grant agreement no. 871336 and ESSENCE project as part of the ATTRACT project funded by the EC under grant agreement no. 777222. We thank N. Kösters, S. Shaffert and I. Levchuk from Siemens Healthineers AG for discussions on imager characterization and data evaluation. We thank the process engineers of Holst Centre's R\&D TFT Pilot Line for the realization of the TFT backplanes. This work is partly financed through the Flexlines project within the Interreg $\mathrm{V}$ programme between The Netherlands and Flanders (Belgium), a cross-border cooperation programme with financial support from the European Regional Development Fund, and co-financed by the Province of Noord-Brabant, The Netherlands. We thank A. Barbash from FAU for the SEM imaging. W.H. thanks the Deutsche Forschungsgemeinschaft (DFG) for financial support via project 404984854 and GRK2495/J.

\section{Author contributions}

S.D., S.F.T., A.v.B. and B.P. performed the electrical characterization under X-ray exposure. J.E.H., S.D. and M.A. fabricated and electrically characterized the soft-sintered $\mathrm{MAPbI}_{3}$ wafers and J.E.H. processed the imagers. S.D. carried out the XRD and SEM measurements. J.M., R.V. and S.S. designed the IGZO TFT backplanes with anchoring grid. S.F.T. and S.D. analysed the results and wrote the manuscript. S.F.T. and O.S. supervised all the activities. M.G.-B. and O.A. prepared the measuring setup and carried out the dielectric and impedance measurements. A.G. and G.G.-B. supervised the dielectric parameter extraction, data fitting and figure drawing. G.G.-B., G.G., A.v.B., H.A., E.M. and W.H. joined the final discussion and read the manuscript drafts.

\section{Competing interests}

The authors declare no competing interests.

\section{Additional information}

Supplementary information The online version contains supplementary material available at https://doi.org/10.1038/s41928-021-00644-3 .

Correspondence and requests for materials should be addressed to Sarah Deumel or Sandro F. Tedde.

Peer review information Nature Electronics thanks John Rowlands and the other, anonymous, reviewer(s) for their contribution to the peer review of this work.

Reprints and permissions information is available at www.nature.com/reprints. Publisher's note Springer Nature remains neutral with regard to jurisdictional claims in published maps and institutional affiliations.

Open Access This article is licensed under a Creative Commons Attribution 4.0 International License, which permits use, sharing, adaptation, distribution and reproduction in any medium or format, as long as you give appropriate credit to the original author(s) and the source, provide a link to the Creative Commons license, and indicate if changes were made. The images or other third party material in this article are included in the article's Creative Commons license, unless indicated otherwise in a credit line to the material. If material is not included in the article's Creative Commons license and your intended use is not permitted by statutory regulation or exceeds the permitted use, you will need to obtain permission directly from the copyright holder. To view a copy of this license, visit http://creativecommons.org/licenses/by/4.0/.

(C) The Author(s) 2021 